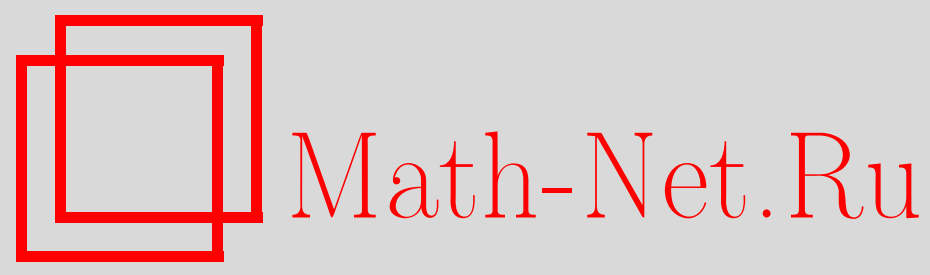

И. И. Шарапудинов, Об асимптотике и весовых оценках полиномов Мейкснера, ортогональных на сетке $\{0, \delta, 2 \delta, \ldots\}$, Матем. заметки, 1997, том 62, выпуск 4, 603-616

DOI: https://doi.org/10.4213/mzm1642

Использование Общероссийского математического портала Math-Net.Ru подразумевает, что вы прочитали и согласны с пользовательским соглашением http://www . mathnet.ru/rus/agreement

Параметры загрузки:

IP: 3.85 .183 .62

26 апреля 2023 г., 10:34:54

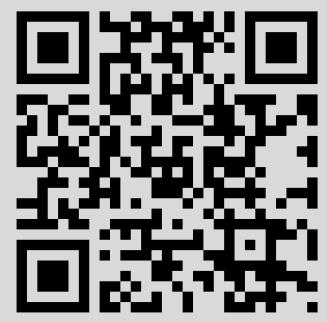




\section{ОБ АСИМПТОТИКЕ И ВЕСОВЫХ ОЦЕНКАХ ПОЛИНОМОВ МЕЙКСНЕРА, ОРТОГОНАЛЬНЫХ НА СЕТКЕ $\{0, \delta, 2 \delta, \ldots\}$}

\section{И.И. Шарапудинов}

Пусть $0<\delta \leqslant 1, N=1 / \delta, 0 \leqslant \alpha$-целое. Для классических полиномов Мейкснера $\mathfrak{M}_{n, N}^{\alpha}(x)$, ортонормированных на сетке $\{0, \delta, 2 \delta, \ldots\}$ с весом $\rho(x)=\left(1-e^{-\delta}\right)^{\alpha} \times$ $\Gamma(N x+\alpha+1) / \Gamma(N x+1)$, получена асимптотическая формула

$$
\mathfrak{M}_{n, N}^{\alpha}(z)=\Lambda_{n}^{\alpha}(z)+v_{n, N}^{\alpha}(z) .
$$

Для остаточного члена $v_{n, N}^{\alpha}(z)$ при $n \leqslant \lambda N$ верна оценка

$$
\left|v_{n, N}^{\alpha}(z)\right|^{2} \leqslant c(\alpha, \lambda) \delta \sum_{k=0}^{n}\left|\Lambda_{k}^{\alpha}(z)\right|^{2}
$$

где $\Lambda_{k}^{\alpha}(x)$ - ортонормированные полиномы Лагерра. Как следствие, получена весовая оценка для полинома Мейкснера $\mathfrak{M}_{n, N}^{\alpha}(x)$ на полуоси $[0, \infty)$.

Библиограффия: 9 названий.

1. Введение. Полиномы Мейкснера [1] относятся к одному из четырех типов классических полиномов, ортогональных на равномерных сетках на действительной оси, которые могут быть представлены с помощью разностного аналога формулы Родрига, хорошо известной в теории полиномов Якоби, Лагерра и Эрмита. Разностные свойства полиномов Мейкснера аналогичны дифференциальным свойствам полиномов Лагерра. В этом смысле полиномы Мейкснера представляют собой дискретный аналог полиномов Лагерра. Следует отметить, что разностные свойства полиномов Мейкснера, равно как и свойства, вытекаюшие из их ортогональности на равномерной сетке, достаточно подробно исследованы, что собственно и составляет классическую теорию этих полиномов. Асимптотическая теория полиномов Мейкснера, несмотря на многочисленные приложения, в которых требуется знание асимптотических свойств этих полиномов, до недавнего времени оставалась неисследованной.

Целенаправленное исследование асимптотических свойств многочленов Мейкснера начато в работах автора [2]-[5], в которых по ряду вопросов удалось получить результаты, близкие к окончательным. В настоящей работе мы продолжаем исследование асимптотических свойств полиномов Мейкснера на комплексной плоскости и на полуоси $[0, \infty)$. Как следствие полученных асимптотических формул, устанавливаем весовую оценку для полиномов Мейкснера на полуоси $[0, \infty)$.

Работа выполнена при финансовой поддержке Российского фонда фундаментальных исследований, грант № 95-01-01526. 
2. Некоторые формулы для полиномов Мейкснера. Пусть $0<q<1, \alpha>-1$,

$$
\mu(x)=\mu(x ; \alpha, q)=q^{x} \frac{\Gamma(x+\alpha+1)}{\Gamma(x+1)}(1-q)^{\alpha+1} .
$$

Через $M_{n}^{\alpha}(x)=M_{n}^{\alpha}(x, q)$ обозначим классические полиномы Мейкснера, нормированные условием

$$
M_{n}^{\alpha}(0)=\left(\begin{array}{c}
n+\alpha \\
n
\end{array}\right) .
$$

Нам понадобятся в дальнейшем следующие формулы [1]: соотношение ортогональности

$$
\sum_{x=0}^{\infty} \mu(x) M_{n}^{\alpha}(x) M_{m}^{\alpha}(x)=\delta_{n m} h_{n, q}^{\alpha},
$$

где $\delta_{n m}-$ символ Кронекера,

$$
h_{n, q}^{\alpha}=q^{-n}\left(\begin{array}{c}
n+\alpha \\
n
\end{array}\right) \Gamma(\alpha+1)
$$

явный вид полиномов Мейкснера

$$
M_{n}^{\alpha}(x, q)=\left(\begin{array}{c}
n+\alpha \\
n
\end{array}\right) \sum_{k=0}^{n} \frac{n^{[k]} x^{[k]}}{(\alpha+1)_{k} k !}\left(1-\frac{1}{q}\right)^{k}
$$

где $a^{[0]}=(a)_{0}=1, a^{[k]}=a \cdots(a-k+1),(a)_{k}=a \cdots(a+k-1)$.

Кроме того, будем использовать следующие обозначения:

$$
\begin{gathered}
N=\frac{1}{\delta}, \quad \Omega_{\delta}=\{0, \delta, 2 \delta, \ldots\} \\
\mathfrak{M}_{n, N}^{\alpha}(x, q)=\left(h_{n, q}^{\alpha}\right)^{-1 / 2} M_{n}^{\alpha}(N x, q), \quad \mathfrak{M}_{n, N}^{\alpha}(x)=\mathfrak{M}_{n, N}^{\alpha}\left(x, e^{-1 / N}\right)
\end{gathered}
$$

3. Некоторые сведения о полиномах Лагерра. Полиномы Лагерра определим с помощью следующего равенства:

$$
L_{n}^{\alpha}(x)=\left(\begin{array}{c}
n+\alpha \\
n
\end{array}\right) \sum_{k=0}^{n} \frac{n^{[k]}(-x)^{k}}{(\alpha+1)_{k} k !}
$$

Хорошо известно [1], что

$$
\int_{0}^{\infty} e^{-x} x^{\alpha} L_{n}^{\alpha}(x) L_{m}^{\alpha}(x) d x=h_{n}^{\alpha} \delta_{n m}
$$

где

$$
h_{n}^{\alpha}=\Gamma(\alpha+1)\left(\begin{array}{c}
n+\alpha \\
n
\end{array}\right) .
$$


Далее, имеют место следующие равенства:

$$
\begin{gathered}
L_{n}^{\alpha}(x)=\frac{1}{n !} e^{x} x^{-\alpha} \frac{d^{n}}{d x^{n}}\left(x^{n+\alpha} e^{-x}\right), \\
L_{n+1}^{\alpha}(x)=L_{n+1}^{\alpha-1}(x)+L_{n}^{\alpha}(x), \\
\frac{d}{d x} L_{n}^{\alpha}(x)=-L_{n-1}^{\alpha+1}(x), \\
K_{n}^{\alpha}(x, y)=\sum_{\nu=0}^{n}\left(\begin{array}{c}
\nu+\alpha \\
\nu
\end{array}\right) L_{\nu}^{\alpha}(x) L_{\nu}^{\alpha}(y) \\
=\frac{(n+1) !}{\Gamma(n+\alpha+1)} \frac{L_{n}^{\alpha}(x) L_{n+1}^{\alpha}(y)-L_{n}^{\alpha}(y) L_{n+1}^{\alpha}(x)}{x-y}, \quad 1 \leqslant k \leqslant n, \\
L_{n}^{-k}(x)=(-x)^{k} \frac{(n-k) !}{n !} L_{n-k}^{k}(x), \quad 1 \leqslant
\end{gathered}
$$

$k$ - целое. Пусть $a_{n}=4 n+2 \alpha+2$,

$$
A_{n}^{\alpha}(x)= \begin{cases}e^{x / 2} a_{n}^{\alpha}, & \text { если } 0 \leqslant x \leqslant 1 / a_{n}, \\ e^{x / 2} a_{n}^{\alpha / 2-1 / 4} x^{-\alpha / 2-1 / 4}, & \text { если } 1 / a_{n}<x \leqslant a_{n} / 2, \\ e^{x / 2}\left(a_{n}\left(a_{n}^{1 / 3}+\left|x-a_{n}\right|\right)\right)^{-1 / 4}, & \text { если } a_{n} / 2<x \leqslant 3 a_{n} / 2, \\ e^{x / 4}, & \text { если } 3 a_{n} / 2<x .\end{cases}
$$

Тогда справедлива [6], [7] оценка

$$
\left|L_{n}^{\alpha}(x)\right| \leqslant c(\alpha) A_{n}^{\alpha}(x), \quad 0 \leqslant x<\infty, \quad \alpha>-1
$$

(здесь и далее, $c(\alpha, \beta, \ldots, \gamma), c_{k}(\alpha, \beta, \ldots, \gamma)$ есть положительные постоянные, зависящие лишь от указанных параметров, вообще говоря, различные в разных местах, например, допускаются записи вида $c(\alpha)<c(\alpha))$.

Пусть $\alpha>-1, \sigma_{n}(x)$ - произвольный полином, подчиненный условию

$$
\int_{0}^{\infty} e^{-x} x^{\alpha}\left|\sigma_{n}(x)\right|^{2} d x \leqslant \theta
$$

Тогда для любого комплексного числа $z$ имеет место неравенство (см. [8, с. 189])

$$
\left|\sigma_{n}(z)\right|^{2} \leqslant \theta \sum_{\nu=0}^{n}\left|\Lambda_{\nu}^{\alpha}(z)\right|^{2}
$$

где

$$
\Lambda_{n}^{\alpha}(z)=\left(h_{n}^{\alpha}\right)^{-1 / 2} L_{n}^{\alpha}(x)
$$


4. О числах Стирлинга и Бернулли. Формула суммирования Эйлера. Положим

$$
x^{[n]}=x(x-1) \cdots(x-n+1)=\sum_{k=0}^{n} s(n, k) x^{k} .
$$

Числа $s(n, k)(k=0,1, \ldots, n)$ в правой части равенства (18) назьваются числами Стирлинга первого рода. В работе автора [9] установлена оценка

$$
|s(n, k)| \leqslant\left(\begin{array}{l}
n \\
k
\end{array}\right) n^{n-k}, \quad 0 \leqslant k \leqslant n .
$$

Числа Бернулли $B_{\nu}(\nu=0,1, \ldots)$ определяются с помощью следующего равенства:

$$
\frac{t}{e^{t}-1}=\sum_{\nu=0}^{\infty} \frac{B_{\nu}}{\nu !} t^{\nu}, \quad|t|<2 \pi
$$

В частности, $B_{0}=1, B_{1}=-1 / 2, B_{2}=1 / 6, B_{2 k+1}=0(k=1,2, \ldots)$. Имеет место оценка

$$
\left|B_{2 k}\right| \leqslant \frac{2(2 k) !}{(2 \pi)^{2 k}\left(1-2^{1-2 k}\right)}, \quad k=1,2, \ldots .
$$

Пусть $F(x)$ имеет $2 n$ непрерьвных производных на сегменте $[a, b]$. Разделим этот сегмент на $m$ равных частей, и пусть $h=(b-a) / m$. Тогда имеет место равенство

$$
\begin{aligned}
\int_{a}^{b} F(x) d x= & h \sum_{k=0}^{m} F(a+k h)-\frac{h}{2}(F(b)+F(a)) \\
& +\sum_{k=1}^{n-1} \frac{h^{2 k}}{(2 k) !} B_{2 k}\left(F^{(2 k-1)}(a)-F^{(2 k-1)}(b)\right) \\
& -\frac{h^{n+1}}{(2 n) !} B_{2 n} \sum_{k=0}^{m-1} F^{(2 n)}(a+k h+\theta h), \quad 0<\theta<1,
\end{aligned}
$$

назьваемое формулой суммирования Эйлера.

Пусть $f_{r}(x)$ - алгебраический полином степени $r, F(x)=e^{-x} f_{r}(x), 0<h \leqslant 1$. Тогда из (21) вытекает равенство

$$
\int_{0}^{\infty} F(x) d x=h \sum_{j=0}^{\infty} F(j h)-\frac{h}{2} F(0)+\sum_{k=1}^{\infty} \frac{h^{2 k} B_{2 k}}{(2 k) !} F^{(2 k-1)}(0)
$$

которое без доказательства приводилось в работе [5]. Дадим подробное доказательство равенства (22). Пусть

$$
f_{r}(x)=\alpha_{0}+\alpha_{1} x+\cdots+\alpha_{r} x^{r}, \quad\left\|f_{r}\right\|=\left|\alpha_{0}\right|+\cdots+\left|\alpha_{r}\right| .
$$

Тогда

$$
f_{r}^{(k)}(x)=\alpha_{k} k !+\alpha_{k+1}(k+1)^{[k]} x+\cdots+\alpha_{r} r^{[k]} x^{r-k}
$$


и, стало быть, при $k \leqslant r$

$$
\left|f_{r}^{(k)}(x)\right| \leqslant\left\|f_{r}\right\|(|x|+1)^{r-k} r^{[k]} .
$$

С другой стороны, в силу формулы Лейбнища

$$
F^{(l)}(x)=\left(e^{-x} f_{r}(x)\right)^{(l)}=e^{-x} \sum_{\nu=0}^{r}(-1)^{l-\nu}\left(\begin{array}{l}
l \\
\nu
\end{array}\right) f_{r}^{(\nu)}(x)
$$

(напомним, что $\left(\begin{array}{l}l \\ \nu\end{array}\right)=0$, если $\left.\nu>l\right)$. Из $(23)$ и $(24)$ находим для $l \geqslant 1$

$$
\begin{aligned}
\left|F^{(l)}(x)\right| & \leqslant e^{-x}\left\|f_{r}\right\| \sum_{\nu=0}^{r} \frac{l^{[\nu]}}{\nu !} r^{[\nu]}(|x|+1)^{r-\nu} \leqslant r ! l^{[r]}\left\|f_{r}\right\|(|x|+1)^{r} e^{-x} \sum_{\nu=0}^{r} \frac{1}{\nu !} \\
& \leqslant r ! e^{-x+1} l^{r}\left\|f_{r}\right\|(|x|+1)^{r} .
\end{aligned}
$$

Неравенство (25) в сочетании с $(20)$ для $0<h \leqslant 1$ дает

$$
\begin{aligned}
\left|\frac{h^{n+1} B_{2 n}}{(2 n) !} \sum_{k=0}^{m-1} F^{(2 n)}(k h+\theta h)\right| & \leqslant \frac{r !(2 n)^{r}\left\|f_{r}\right\|}{(2 \pi)^{2 n}} \frac{2 e}{1-2^{1-2 n}} \sum_{k=0}^{\infty}((k+1) h+1)^{r} e^{-k h} \\
& \leqslant c(r)\left\|f_{r}\right\| \frac{(2 n)^{r}}{(2 \pi)^{2 n}} \rightarrow 0, \quad n \rightarrow \infty
\end{aligned}
$$

Полагая $a=0, b=Q h$, из (21) и (26) выводим

$$
\begin{aligned}
\int_{0}^{Q h} F(x) d x= & h \sum_{k=0}^{Q} F(k h)-\frac{h}{2}(F(Q h)+F(0)) \\
& +\sum_{\nu=1}^{\infty} h^{2 \nu} \frac{B_{2 \nu}}{(2 \nu) !}\left(F^{(2 \nu-1)}(0)-F^{(2 \nu-1)}(Q h)\right) .
\end{aligned}
$$

(Здесь мы вновь воспользовались оценками (20) и (25), из которых вытекает, что ряд в правой части равенства (27) абсолютно сходится при $0<h \leqslant 1$.) Далее, из (20) и $(25)$ имеем

$$
\begin{aligned}
\sum_{\nu=1}^{\infty} h^{2 \nu}\left|\frac{B_{2 \nu}}{(2 \nu) !} F^{(2 \nu-1)}(Q h)\right| & \leqslant r !\left\|f_{r}\right\|(Q h+1)^{r} e^{-Q h+1} \sum_{\nu=1}^{\infty} \frac{(2 \nu-1)^{r} h^{2 \nu} B_{2 \nu}}{(2 \nu) !} \\
& \leqslant c(r)\left\|f_{r}\right\|(Q h+1)^{r} e^{-Q h} \rightarrow 0, \quad Q \rightarrow \infty .
\end{aligned}
$$

Поэтому, переходя к пределу при $Q \rightarrow \infty$, из (27) получаем (22).

5. Об оценке производных полинома Мейкснера $M_{n}^{\alpha}\left(x, e^{-\delta}\right)$. Здесь мы установим одно вспомогательное неравенство для производной порядка $r \leqslant n$ полинома Мейкснера $M_{n}^{\alpha}(x, q)$ при $x=0, \alpha \geqslant 0, q=e^{-\delta}$ и получим некоторые следствия из него. Полагая $p=q^{-1}-1$, из (4) имеем

$$
\begin{aligned}
\left(M_{n}^{\alpha}(0, q)\right)^{(r)} & =\left(\begin{array}{c}
n+\alpha \\
n
\end{array}\right)\left(\sum_{\nu=0}^{n} \frac{n^{[\nu]} x^{[\nu]}}{(\alpha+1)_{\nu} \nu !}(-p)^{\nu}\right)_{x=0}^{(r)} \\
& =\left(\begin{array}{c}
n+\alpha \\
n
\end{array}\right) \sum_{\nu=r}^{n} \frac{n^{[\nu]}\left(x^{[\nu]}\right)_{x=0}^{(r)}}{(\alpha+1)_{\nu} \nu !}(-p)^{\nu}
\end{aligned}
$$


Так как в силу (18)

$$
\left(x^{[\nu]}\right)^{(r)}=\sum_{j=r}^{\nu} j^{[r]} s(\nu, j) x^{j-r}
$$

то

$$
\left(x^{[\nu]}\right)_{x=0}^{(r)}=s(\nu, r) r ! .
$$

Сопоставляя $(28)$ и $(29)$, находим $(r \leqslant n)$

$$
\left(M_{n}^{\alpha}(0, q)\right)^{(r)}=\left(\begin{array}{c}
n+\alpha \\
n
\end{array}\right) \sum_{\nu=r}^{n} \frac{n^{[\nu]} s(\nu, r) r !}{(\alpha+1)_{\nu} \nu !}(-p)^{\nu}
$$

Воспользуемся оценкой (19). Тогда из (30), воспользовавшись формулой Стирлинга, имеем

$$
\begin{aligned}
\left|\left(M_{n}^{\alpha}(0, q)\right)^{(r)}\right| & \leqslant \frac{\Gamma(n+\alpha+1)}{n !}(n p)^{r} \sum_{\nu=r}^{n} \frac{\nu^{\nu-r}(n p)^{\nu-r}}{\Gamma(\nu+\alpha+1)(\nu-r) !} \\
& \leqslant(n p)^{r} \frac{\Gamma(n+\alpha+1)}{(2 \pi)^{1 / 2} n !} \sum_{\nu=r}^{n} \frac{\nu^{\nu-r}(n p)^{\nu-r} e^{\nu+\alpha+1}}{(\nu+\alpha+1)^{\nu+\alpha}(\nu-r) !} \\
& \leqslant(n p)^{r} \frac{\Gamma(n+\alpha+1) e^{r+\alpha+1}}{(2 \pi)^{1 / 2} n !(r+\alpha+1)^{r+\alpha}} \sum_{\nu=r}^{n} \frac{(e n p)^{\nu-r}}{(\nu-r) !} .
\end{aligned}
$$

Отсюда мы заключаем, что справедлива следующая

Лемма 5.1. Пусть $\alpha \geqslant 0, p=q^{-1}-1,0 \leqslant r-$ произвольное иелое число. Тогда

$$
\left|\left(M_{n}^{\alpha}(0, q)\right)^{(r)}\right| \leqslant \frac{\Gamma(n+\alpha+1)(n p)^{r}}{(2 \pi)^{1 / 2} n !(r+\alpha+1)^{r+\alpha}} e^{r+\alpha+p n e+1} .
$$

В самом деле, справедливость леммы 5.1 при $0 \leqslant r \leqslant n$ следует из (31), а при $r>n$ очевидна.

Ниже потребуются оценки для производных полиномов $\left(M_{n}^{\alpha}(x, q)\right)^{2}$ при $x=0$.

Лемма 5.2. Пусть $\alpha \geqslant 0, p=q^{-1}-1,0 \leqslant m-$ произвольное иелое число. Тогда

$$
\left|\left(\left(M_{n}^{\alpha}(x, q)\right)^{2}\right)_{x=0}^{(m)}\right| \leqslant\left(\frac{\Gamma(n+\alpha+1)}{n !}\right)^{2} \frac{4^{\alpha}}{2 \pi} \frac{e^{m+2(1+\alpha)+2 e n p}}{(m+2 \alpha+2)^{m+2 \alpha}}(4 n p)^{m} .
$$

ДокАЗАТЕЛЬСТВо. Воспользовавшись формулой Лейбница, имеем

$$
\left(\left(M_{n}^{\alpha}(x, q)\right)^{2}\right)_{x=0}^{(m)}=\sum_{l=0}^{m}\left(\begin{array}{c}
m \\
l
\end{array}\right)\left(M_{n}^{\alpha}(x, q)\right)_{x=0}^{(l)}\left(M_{n}^{\alpha}(x, q)\right)_{x=0}^{(m-l)}
$$


Отсюда и из леммы 5.1 следует, что

$$
\begin{aligned}
\left|\left(\left(M_{n}^{\alpha}(x, q)\right)^{2}\right)_{x=0}^{(m)}\right| \leqslant & \frac{1}{2 \pi} e^{m+2(1+\alpha)+2 e n p}(n p)^{m}\left(\frac{\Gamma(n+\alpha+1)}{n !}\right)^{2} \\
& \times \sum_{l=0}^{m} \frac{\left(\begin{array}{c}
m \\
l
\end{array}\right)}{(l+\alpha+1)^{l+\alpha}(m-l+\alpha+1)^{m-l+\alpha}} \\
\leqslant & \left(\frac{\Gamma(n+\alpha+1)}{n !}\right)^{2} \frac{4^{\alpha}}{2 \pi} \frac{e^{m+2(1+\alpha)+2 e n p}}{(m+2 \alpha+2)^{m+2 \alpha}}(2 n p)^{m} \sum_{l=0}^{m}\left(\begin{array}{c}
m \\
l
\end{array}\right),
\end{aligned}
$$

так как минимум функции $\varphi(l)=(l+\alpha+1)^{l+\alpha}(m-l+\alpha+1)^{m-l+\alpha}(0 \leqslant l \leqslant m)$ равен $\varphi(m / 2)$. Поскольку

$$
\sum_{l=0}^{m}\left(\begin{array}{c}
m \\
l
\end{array}\right)=2^{m}
$$

то утверждение леммы 5.2 непосредственно вытекает из (32).

$$
\begin{gathered}
\text { Пусть } \delta>0, N=1 / \delta, q=e^{-\delta}, \Omega_{\delta}=\{0, \delta, 2 \delta, \ldots\} \\
\rho(x)=\rho(x, \alpha, \delta)=\mu\left(\frac{x}{\delta}, \alpha, q\right), \\
\mathfrak{M}_{n}^{\alpha}(x)=\mathfrak{M}_{n, N}^{\alpha}(x, q)=\left(h_{n, q}\right)^{-1 / 2} M_{n}^{\alpha}\left(\frac{x}{\delta}, q\right), \quad n=0,1, \ldots
\end{gathered}
$$

Тогда из (2) имеем

$$
\sum_{x \in \Omega_{\delta}} \mathfrak{M}_{n}^{\alpha}(x) \mathfrak{M}_{k}^{\alpha}(x) \rho(x)=\delta_{n k}
$$

другими словами, полиномы $\mathfrak{M}_{n}^{\alpha}(x)(n=0,1, \ldots)$ образуют ортонормированную систему на множестве $\Omega_{\delta}$ с весом $\rho(x)$.

ЛЕмма 5.3. В условиях леммы 5.2 имеет место оценка

$$
\left|\left(\left(\mathfrak{M}_{n, N}^{\alpha}(x, q)\right)^{2}\right)_{x=0}^{(m)}\right| \leqslant \frac{\Gamma(n+\alpha+1)}{n !} q^{n} \frac{4^{\alpha}}{2 \pi} \frac{e^{m+2(1+\alpha)+2 e n p}}{(m+2 \alpha+2)^{m+2 \alpha}}\left(\frac{4 p n}{\delta}\right)^{m} .
$$

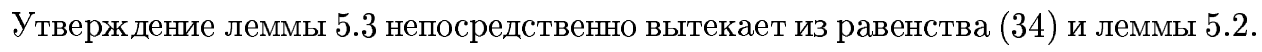
Положим $\varkappa(x)=x^{\alpha} e^{-x}$,

$$
d_{k}=\left(\varkappa(x)\left(\mathfrak{M}_{n}^{\alpha}(x)\right)^{2}\right)_{x=0}^{(2 k-1)}
$$

Величина $d_{k}$ играет ключевую роль при оценке остаточного члена асимптотической формулы для полиномов Мейкснера $\mathfrak{M}_{n}^{\alpha}(x)$. Нам понадобится 
Лемма 5.4. Пусть $0 \leqslant \alpha-$ иелое. Тогда

$$
\left|d_{k}\right| \leqslant \frac{1}{8 \pi} \exp ((1+\alpha)(1-\delta)-n \delta+2 e n p) \frac{(n+\alpha) !}{n ! n^{\alpha+1}}(2 k-1)^{\alpha} A,
$$

əде

$$
A=\frac{\left(16 e^{1+\delta} n\right)^{k}}{4(2+2 \alpha)^{2 \alpha}}+\frac{1}{\alpha !} \exp \left(\frac{2 k-1}{4 n \exp (1+\delta)}\right)\left(\frac{16 e^{2(1+\delta)} n^{2}}{k+2+\alpha}\right)^{k}
$$

ДокАЗАТЕЛЬСтво. Если $0 \leqslant \alpha-$ целое, то из (36) имеем

$$
d_{k}=\sum_{j=\alpha}^{2 k-1}\left(\begin{array}{c}
2 k-1 \\
j
\end{array}\right) \varkappa^{(j)}(0)\left(\left(\mathfrak{M}_{n, N}^{\alpha}(0)\right)^{2}\right)^{(2 k-1-j)} .
$$

Отсюда и из леммы 5.3 находим $\left(q=e^{-\delta}\right)$

$$
\left|d_{k}\right| \leqslant \frac{(n+\alpha) !}{2 \pi n !} 4^{\alpha} e^{2(1+\alpha)-n \delta+2 e n p} B,
$$

где

$$
B=\sum_{j=\alpha}^{2 k-1}\left(\begin{array}{c}
2 k-1 \\
j
\end{array}\right)\left(\frac{4 e n p}{\delta}\right)^{2 k-1-j} \frac{\left|\varkappa^{(j)}(0)\right|}{(2 k+1+2 \alpha-j)^{2 k+2 \alpha-1-j}} .
$$

Далее, из (8), (12) и (5) имеем

$$
\begin{aligned}
\varkappa^{(j)}(0) & =\left.\frac{d^{j}}{d x^{j}}\left(x^{\alpha} e^{-x}\right)\right|_{x=0}=\left.\frac{d^{j}}{d x^{j}}\left(x^{j+\alpha-j} e^{-x}\right)\right|_{x=0}=\left.x^{-j+\alpha} e^{x} j ! L_{j}^{\alpha-j}(x)\right|_{x=0} \\
& =\left.(-1)^{j-\alpha} e^{x} j ! \frac{\alpha !}{j !} L_{\alpha}^{j-\alpha}(x)\right|_{x=0}=(-1)^{j-\alpha} j^{[\alpha]}, \quad j \geqslant \alpha,
\end{aligned}
$$

и $p / \delta<e^{\delta}$, поэтому из $(37)$ находим

$$
\left|d_{k}\right| \leqslant \frac{(n+\alpha) !}{2 \pi n !} 4^{\alpha} e^{2(1+\alpha)-n \delta+2 e n p}\left(4 n e^{1+\delta}\right)^{2 k-1-\alpha} S,
$$

где

$$
S=\sum_{j=\alpha}^{2 k-1}\left(\begin{array}{c}
2 k-1 \\
j
\end{array}\right) \frac{j^{[\alpha]}\left(4 n e^{1+\delta}\right)^{\alpha-j}}{(2 k+1+2 \alpha-j)^{2 k-1+2 \alpha-j}} .
$$

Разобьем сумму (39) по схеме

$$
S=\sum_{j=\alpha}^{k+\alpha-1}+\sum_{j=k+\alpha}^{2 k-1}=S_{1}+S_{2}
$$


Тогда имеем

$$
\begin{aligned}
S_{1} & \leqslant \sum_{j=\alpha}^{k+\alpha+1} \frac{(2 k-1)^{[j]}}{j !\left(4 n e^{1+\delta}\right)^{j-\alpha}}\left(\frac{k+\alpha}{k+2+\alpha}\right)^{\alpha} \frac{1}{(k+2+\alpha)^{k}} \\
& <\frac{(2 k-1)^{[\alpha]}}{\alpha !(k+2+\alpha)^{k}} \exp \left(\frac{2 k-1}{4 n \exp (1+\delta)}\right) \\
S_{2} & =\sum_{j=k+\alpha}^{2 k-1}\left(\begin{array}{c}
2 k-1 \\
j
\end{array}\right) \frac{j^{[\alpha]}\left(4 e^{1+\delta} n\right)^{\alpha-j}}{(2 k+1+2 \alpha-j)^{2 k-1-j+2 \alpha}} \\
& \leqslant \frac{(2 k-1)^{[\alpha]}}{\left(4 e^{1+\delta} n\right)^{k}} \sum_{j=k+\alpha}^{2 k-1}\left(\begin{array}{c}
2 k-1 \\
j
\end{array}\right) \frac{1}{(2 k+1+2 \alpha-j)^{2 \alpha}} \\
& \leqslant \frac{(2 k-1)^{[\alpha]}}{\left(4 e^{1+\delta} n\right)^{k}(2 \alpha+2)^{2 \alpha}} \sum_{j=k+\alpha}^{2 k-1}\left(\begin{array}{c}
2 k-1 \\
j
\end{array}\right) \\
& \leqslant \frac{(2 k-1)^{\alpha} 2^{2 k-2}}{(2 \alpha+2)^{2 \alpha}\left(4 e^{1+\delta} n\right)^{k}}=\frac{(2 k-1)^{[\alpha]}}{4(2 \alpha+2)^{2 \alpha}}\left(e^{1+\delta} n\right)^{-k}
\end{aligned}
$$

Сопоставляя (38)-(41), находим

$$
\begin{aligned}
\left|d_{k}\right| \leqslant & \frac{(n+\alpha) ! 4^{\alpha}}{2 \pi n !} e^{2(1+\alpha)-n \delta+2 e n p}\left(4 n e^{1+\delta}\right)^{-1-\delta}\left(4 n e^{1+\delta}\right)^{2 k} \\
& \times\left(\frac{(2 k-1)^{[\alpha]}}{4(2 \alpha+2)^{2 \alpha}}\left(e^{1+\delta} n\right)^{-k}+\frac{(2 k-1)^{[\alpha]}}{\alpha !(k+2+\alpha)^{k}} \exp \left(\frac{2 k-1}{4 n e^{1+\delta}}\right)\right) \\
\leqslant & \frac{1}{8 \pi} e^{(1+\alpha)(1-\delta)-n \delta+2 e n p} \frac{(n+\alpha) !(2 k-1)^{\alpha}}{n^{1+\alpha} n !} \\
& \times\left(\frac{\left(16 e^{1+\delta} n\right)^{k}}{4(2 \alpha+2)^{2 \alpha}}+\frac{1}{\alpha !}\left(\frac{16 e^{2(1+\delta)} n^{2}}{k+2+\alpha}\right)^{k} \exp \left(\frac{2 k-1}{4 n e^{1+\delta}}\right)\right) .
\end{aligned}
$$

Лемма 5.4 доказана.

6. Асимптотика полиномов Мейкснера $\mathfrak{M}_{n, N}^{\alpha}(x)$ при целых $\alpha$. В случае целого $\alpha \geqslant 0$ установим асимптотическую формулу для полиномов Мейкснера $\mathfrak{M}_{n, N}^{\alpha}(z)$ на всей комплексной плоскости при условии $n=O(N)$.

Теорема 6.1. Пусть $0 \leqslant \alpha-$ иелое, $\lambda, \delta>0, N=1 / \delta, z-$ произвольное комплексное число. Тогда имеет место асимптотическая формула

$$
\mathfrak{M}_{n, N}^{\alpha}(z)=\Lambda_{n}^{\alpha}(z)+v_{n, N}^{\alpha}(z)
$$

в которой для остаточного члена $v_{n, N}^{\alpha}(z)$ при $0<\delta \leqslant 1,0 \leqslant n \leqslant \lambda N$ справедлива оченка

$$
\left|v_{n, N}^{\alpha}(z)\right| \leqslant c\left(\delta \sum_{k=0}^{n}\left|\Lambda_{k}^{\alpha}(z)\right|^{2}\right)^{1 / 2}
$$

əде $c=c(\alpha, \lambda), \Lambda_{k}^{\alpha}(z)$ - ортонормированные полиномы Лагерра, определенные равенством (17). 
ДоКАЗАТЕЛЬСтво. Заметим, что $v_{0, n}^{\alpha}(z)=0$ для произвольного комплексного $z$, поэтому мы можем считать, что $n \geqslant 1$. Оценим следующий интеграл:

$$
\begin{aligned}
\int_{0}^{\infty} & \varkappa(x)\left(v_{n, N}^{\alpha}(x)\right)^{2} d x \\
= & \int_{0}^{\infty} \varkappa(x)\left(\Lambda_{n}^{\alpha}(x)\right)^{2} d x+\int_{0}^{\infty} \varkappa(x)\left(\mathfrak{M}_{n, N}^{\alpha}(x)\right)^{2} d x-2 \int_{0}^{\infty} \varkappa(x) \Lambda_{n}^{\alpha}(x) \mathfrak{M}_{n, N}^{\alpha}(x) d x \\
= & J_{1}+J_{2}-J_{3} .
\end{aligned}
$$

Из (6) и (17) следует, что $J_{1}=1$. Далее, $J_{3}=2 k_{n, N} / k_{n}$, где $k_{n, N}$ и $k_{n}$ - старшие коэффициенты полиномов Мейкснера $\mathfrak{M}_{n, N}^{\alpha}(z)$ и Лагерра $\Lambda_{n}^{\alpha}(z)$ соответственно. Сопоставляя (5), (7), (17) с (3), (4), (34), находим $\left(q=e^{-\delta}, p=e^{\delta}-1\right)$

$$
\frac{k_{n, N}}{k_{n}}=\left(\frac{h_{n}^{\alpha}}{h_{n, q}^{\alpha}}\right)^{1 / 2}\left(\delta^{-1} p\right)^{n}=\left(\delta^{-1}\left(e^{\delta / 2}-e^{-\delta / 2}\right)\right)^{n} .
$$

Так как

$$
\frac{1}{\delta}\left(e^{\delta / 2}-e^{-\delta / 2}\right)=1+\frac{(\delta / 2)^{2}}{3 !}+\frac{(\delta / 2)^{4}}{5 !}+\cdots>1,
$$

то из (44) имеем

$$
J_{3}=2 \frac{k_{n, N}}{k_{n}}>2 .
$$

Рассмотрим интеграл $J_{2}$. Чтобы его оценить, воспользуемся равенством (22). Это дает

$$
\begin{aligned}
J_{2} & =\int_{0}^{\infty} \varkappa(x)\left(\mathfrak{M}_{n, N}^{\alpha}(x)\right)^{2} d x \\
& =\delta \sum_{j=0}^{\infty} \varkappa(j \delta)\left(\mathfrak{M}_{n, N}^{\alpha}(j \delta)\right)^{2}-\frac{\delta}{2}\left(\mathfrak{M}_{n, N}^{\alpha}(0)\right)^{2} \varkappa(0)+\sum_{k=1}^{\infty} \frac{B_{2 k}}{(2 k) !} \delta^{2 k} d_{k},
\end{aligned}
$$

где $d_{k}$ - число, определенное равенством (36).

Пусть $0<\delta \leqslant 1,0 \leqslant x<\infty$. Из (1), (33) при $q=e^{-\delta}$ имеем

$$
\begin{aligned}
\frac{\delta \varkappa(x)}{\rho(x)} & =\frac{\delta x^{\alpha} e^{-x}}{\rho(x)}=\left(\frac{\delta}{1-e^{-\delta}}\right)^{\alpha+1} \frac{x^{\alpha}}{(x+\delta) \cdots(x+\alpha \delta)} \\
& <\left(\frac{\delta}{1-e^{-\delta}}\right)^{\alpha+1}<e^{\delta(\alpha+1)}<1+\delta(\alpha+1) e^{\delta(\alpha+1)} .
\end{aligned}
$$

Из (35) и (46) выводим

$$
\delta \sum_{j=0}^{\infty} \varkappa(j \delta)\left(\mathfrak{M}_{n, N}^{\alpha}(j \delta)\right)^{2}<1+\delta(1+\alpha) e^{\delta(1+\alpha)},
$$

отсюда и из (46), в свою очередь, имеем

$$
J_{2}<1+\delta(1+\alpha) e^{\delta(1+\alpha)}+\sum_{k=1}^{\infty} \frac{B_{2 k}}{(2 k) !} \delta^{2 k} d_{k} .
$$


Сопоставляя (43), (45) и (47), получаем

$$
\int_{0}^{\infty} \varkappa(x)\left(v_{n, N}^{\alpha}(x)\right)^{2} d x<\delta(1+\alpha) e^{\delta(1+\alpha)}+\sum_{k=1}^{\infty} \frac{B_{2 k}}{(2 k) !} \delta^{2 k} d_{k}
$$

Если теперь $0<\delta \leqslant 1$ и $n \leqslant \lambda N$, то из леммы 5.4 и оценки $(20)$ выводим

$$
\begin{aligned}
& \sum_{k=1}^{\infty} \frac{\left|B_{2 k}\right|}{(2 k) !} \delta^{2 k}\left|d_{k}\right| \leqslant c(\alpha, \lambda) \frac{1}{n} \sum_{k=1}^{\infty} k^{\alpha}\left(\frac{\delta}{2 \pi}\right)^{2 k}\left(\left(16 e^{1+\delta} n\right)^{k}+\left(\frac{16 e^{2(1+\delta)} n^{2} e}{k+2+\alpha}\right)^{k}\right) \\
& \quad=c(\alpha, \lambda) \frac{1}{n} \sum_{k=1}^{\infty} \delta^{k}\left(\frac{16 e^{1+\delta}}{4 \pi^{2}} n \delta\right)^{k} k^{\alpha}+c(\alpha, \lambda) \frac{1}{n} \sum_{k=1}^{\infty}\left(\frac{16 e^{2(1+\delta)+1} n^{2} \delta^{2}}{4 \pi^{2}(k+2+\alpha)}\right)^{k} k^{\alpha} \\
& \quad \leqslant c_{1}(\alpha, \lambda) \frac{n \delta^{2}}{n} \sum_{k=1}^{\infty}\left(\frac{16 e^{1+\delta} \lambda \delta}{4 \pi^{2}}\right)^{k-1} k^{\alpha}+c_{2}(\alpha, \lambda) \frac{(n \delta)^{2}}{n} \sum_{k=1}^{\infty}\left(\frac{16 e^{2(1+\delta)+1} \lambda^{2}}{4 \pi^{2}(k+2+\alpha)}\right)^{k-1} k^{\alpha} .
\end{aligned}
$$

Последний ряд в (49) сходится, а что касается предпоследнего ряда, то он также сходится, если $\delta$ выбрать настолько малым, что

$$
\frac{16 e^{2} \lambda \delta}{4 \pi^{2}} \leqslant \frac{1}{2}
$$

Тогда из (48) и (49) заключаем, что

$$
\int_{0}^{\infty} \varkappa(x)\left(v_{n, N}^{\alpha}(x)\right)^{2} d x \leqslant c(\alpha, \lambda) \delta
$$

если только $\delta$ удовлетворяет неравенству (50). Тем самьм, мы доказали, что для полинома $\sigma_{n}(x)=v_{n, N}^{\alpha}(x)$ имеет место оценка $(15)$, в которой $\theta=c(\alpha, \lambda) \delta$, стало быть, справедливо неравенство (16), т.е.

$$
\left|v_{n, N}^{\alpha}(z)\right|^{2} \leqslant c(\alpha, \lambda) \delta \sum_{\nu=0}^{n}\left|\Lambda_{\nu}^{\alpha}(z)\right|^{2}
$$

Отсюда и вытекает утверждение теоремы 6.1 , соответствующее случаю (50).

Рассмотрим случай, когда $0<\delta_{0} \leqslant \delta \leqslant 1$, где $\delta_{0}$ фиксировано. Положим $n_{0}=\left[\lambda / \delta_{0}\right]$, $n_{\delta}=[\lambda / \delta]$, где [·] - целая часть числа. Ясно, что если $n \leqslant \lambda N$, то $n \leqslant n_{\delta} \leqslant n_{0}$, поэтому достаточно установить оценку (42) при $n \leqslant n_{0}$. Сопоставляя (34) с (4), нетрудно убедиться в том, что

$$
\left(\mathfrak{M}_{n, N}^{\alpha}(x)\right)^{2}=a_{0, n}(\delta)+a_{1, n}(\delta) x+\cdots+a_{2 n, n}(\delta) x^{2 k},
$$

где $a_{k, n}(\delta)$ - непрерывные функции параметра $\delta$ на отрезке $\left[\delta_{0}, 1\right]$. Положим

$$
\begin{aligned}
& \bar{a}=\bar{a}\left(\delta_{0}\right)=\max _{\delta_{0} \leqslant \delta \leqslant 1} \max _{0 \leqslant n \leqslant n_{0}} \max _{0 \leqslant k \leqslant 2 n}\left|a_{k, n}(\delta)\right|, \\
& \bar{b}=\bar{b}\left(\delta_{0}\right)=\max _{0 \leqslant k \leqslant 2 n_{0}} \int_{0}^{\infty} x^{k+\alpha} e^{-x} d x .
\end{aligned}
$$


Тогда имеем

$$
\int_{0}^{\infty} x^{\alpha} e^{-x}\left(\mathfrak{M}_{n, N}^{\alpha}(x)\right)^{2} d x \leqslant\left(2 n_{0}+1\right) \bar{a} \bar{b}
$$

Обратимся теперь к неравенству (16). Полагая $\sigma_{n}(x)=\mathfrak{M}_{n, N}^{\alpha}(x), \theta=\left(2 n_{0}+1\right) \bar{a} \bar{b}$, имеем

$$
\left|\mathfrak{M}_{n, N}^{\alpha}(x)\right| \leqslant\left(\left(2 n_{0}+1\right) \bar{a} \bar{b} \sum_{\nu=0}^{n}\left|\Lambda_{\nu}^{\alpha}(z)\right|^{2}\right)^{1 / 2} .
$$

Так как $\left(2 n_{0}+1\right) \bar{a} \bar{b}$ зависит только от $\alpha, \lambda$ и $\delta_{0}$, то из (51) находим

$$
\left|\mathfrak{M}_{n, N}^{\alpha}(z)\right| \leqslant c\left(\alpha, \lambda, \delta_{0}\right)\left(\sum_{\nu=0}^{n}\left|\Lambda_{\nu}^{\alpha}(z)\right|^{2}\right)^{1 / 2} .
$$

Отсюда для $\delta_{0} \leqslant \delta \leqslant 1$ получаем

$$
\begin{aligned}
\left|v_{n, N}^{\alpha}(z)\right| & =\left|\mathfrak{M}_{n, N}^{\alpha}(z)-\Lambda_{n}^{\alpha}(z)\right| \leqslant\left|\mathfrak{M}_{n, N}^{\alpha}(z)\right|+\left|\Lambda_{n}^{\alpha}(z)\right| \\
& \leqslant c_{1}\left(\alpha, \lambda, \delta_{0}\right)\left(\delta \sum_{\nu=0}^{n}\left|\Lambda_{\nu}^{\alpha}(z)\right|^{2}\right)^{1 / 2} \cdot
\end{aligned}
$$

Положим $\delta_{0}=\pi^{2} /\left(8 e^{2} \lambda\right)$. Тогда для $0<\delta \leqslant \delta_{0}$ вьполнено неравенство (50), и, стало быть, для таких $\delta$ справедливо утверждение теоремы 6.1 . Если же $\delta_{0} \leqslant \delta \leqslant 1$, то верна оценка (52), которая приводит к утверждению теоремы 6.1 для оставшихся $\delta$. Теорема полностью доказана.

7. Весовые оценки полиномов Мейкснера. Перейдем к исследованию поведения полиномов Мейкснера на полуоси $[0, \infty)$. Здесь мы получим весовую оценку для этих полиномов.

Пусть, по-прежнему, $\delta>0, q=e^{-\delta}, N=1 / \delta$. Основным результатом этого пункта является

Теорема 7.1. Пусть $0 \leqslant \alpha-$ иелое, $0<\delta \leqslant 1, \lambda>0$. Тогда имеет место оценка

$$
\left|\mathfrak{M}_{n, N}^{\alpha}(x)\right| \leqslant c n^{-\alpha / 2} A_{n}^{\alpha}(x), \quad 0 \leqslant x<\infty, \quad n \leqslant \lambda N,
$$

в которой $c=c(\alpha, \lambda), A_{n}^{\alpha}(x)-$ функиия, определенная формулой (13).

ДокАЗАТЕЛЬСТво. Обратимся к формуле Кристоффеля-Дарбу (11). В силу (9) ее можно переписать в виде

$$
\begin{aligned}
& K_{n}^{\alpha}(t, x)=\frac{(n+1) !}{\Gamma(n+\alpha+1)(x-t)}\left(L_{n+1}^{\alpha}(x) L_{n+1}^{\alpha-1}(t)-L_{n+1}^{\alpha-1}(x) L_{n+1}^{\alpha}(t)\right) \\
& \quad=\frac{(n+1) !}{\Gamma(n+\alpha+1)}\left(L_{n+1}^{\alpha}(x) \frac{L_{n+1}^{\alpha-1}(x)-L_{n+1}^{\alpha-1}(t)}{t-x}-L_{n+1}^{\alpha-1}(x) \frac{L_{n+1}^{\alpha}(x)-L_{n+1}^{\alpha}(t)}{t-x}\right) .
\end{aligned}
$$

Переходя к пределу при $t \rightarrow x$ и пользуясь равенством $(10)$, имеем

$$
\begin{aligned}
K_{n}^{\alpha}(x, x) & =\sum_{\nu=0}^{n}\left(\Lambda_{\nu}^{\alpha}(x)\right)^{2}=\frac{(n+1) !}{\Gamma(n+\alpha+1)}\left(-L_{n+1}^{\alpha}(x)\left(L_{n+1}^{\alpha-1}(x)\right)^{\prime}+L_{n+1}^{\alpha-1}(x)\left(L_{n+1}^{\alpha}(x)\right)^{\prime}\right) \\
& =\frac{(n+1) !}{\Gamma(n+\alpha+1)}\left(L_{n+1}^{\alpha}(x) L_{n}^{\alpha}(x)-L_{n+1}^{\alpha-1}(x) L_{n}^{\alpha+1}(x)\right) .
\end{aligned}
$$


Воспользуемся теоремой 6.1. Пусть $n \leqslant \lambda N, 0 \leqslant x<\infty, \delta=1 / N$. Тогда из оценки (42) и равенства (53) находим

$$
\left|v_{n, N}^{\alpha}(x)\right| \leqslant c(\alpha, \lambda)\left(\frac{n}{N}\right)^{1 / 2}\left(\begin{array}{c}
n+\alpha \\
n
\end{array}\right)^{-1 / 2}\left(\left|L_{n}^{\alpha}(x) L_{n+1}^{\alpha}(x)\right|+\left|L_{n}^{\alpha+1}(x) L_{n+1}^{\alpha-1}(x)\right|\right)^{1 / 2} .
$$

Кроме того,

$$
\left(\begin{array}{c}
n+\alpha \\
n
\end{array}\right)^{-1 / 2} \leqslant c(\alpha) n^{-\alpha / 2}
$$

поэтому с учетом (17) получаем

$$
\begin{aligned}
\left|\mathfrak{M}_{n, N}^{\alpha}(x)\right| \leqslant & \left|\Lambda_{n}^{\alpha}(x)\right|+\left|v_{n, N}^{\alpha}(x)\right|=\left(\begin{array}{c}
n+\alpha \\
n
\end{array}\right)^{-1 / 2}\left|L_{n}^{\alpha}(x)\right|+\left|v_{n, N}^{\alpha}(x)\right| \\
\leqslant & c(\alpha) n^{-\alpha / 2}\left|L_{n}^{\alpha}(x)\right|+c(\alpha, \lambda) n^{-\alpha / 2} \\
& \quad \times\left(\left|L_{n}^{\alpha}(x) L_{n+1}^{\alpha}(x)\right|+\left|L_{n}^{\alpha+1}(x) L_{n+1}^{\alpha-1}(x)\right|\right)^{1 / 2} \\
\leqslant & c(\alpha, \lambda) n^{-\alpha / 2}\left(\left|L_{n}^{\alpha}(x)\right|+\left(\left|L_{n}^{\alpha}(x) L_{n+1}^{\alpha}(x)\right|+\left|L_{n}^{\alpha+1}(x) L_{n+1}^{\alpha-1}(x)\right|\right)^{1 / 2}\right) .
\end{aligned}
$$

Чтобы оценить полиномы Лагерра, фигурирующие в (54), обратимся к неравенству (14). Из равенства (13) следует, что равномерно относительно $x \in[0, \infty)$

$$
A_{n}^{\alpha}(x) \asymp A_{n+1}^{\alpha}(x), \quad n=1,2, \ldots,
$$

(запись $a_{n} \asymp b_{n}$ означает, что $\left|a_{n}\right| \leqslant c_{1}\left|b_{n}\right|,\left|b_{n}\right| \leqslant c_{2}\left|a_{n}\right|, n=1,2, \ldots$ ). Поэтому из (14) имеем

$$
\left(\left|L_{n}^{\alpha}(x) L_{n+1}^{\alpha}(x)\right|\right)^{1 / 2} \leqslant c(\alpha) A_{n}^{\alpha}(x), \quad 0 \leqslant x<\infty .
$$

Из (13) нетрудно также заметить, что если $\alpha>0$, то

$$
A_{n}^{\alpha+1}(x) A_{n+1}^{\alpha-1}(x) \leqslant c(\alpha)\left(A_{n}^{\alpha}(x)\right)^{2}, \quad x \geqslant 0,
$$

поэтому в этом случае

$$
\left(\left|L_{n}^{\alpha+1}(x) L_{n+1}^{\alpha-1}(x)\right|\right)^{1 / 2} \leqslant c(\alpha) A_{n}^{\alpha}(x), \quad x \geqslant 0 .
$$

Если $\alpha=0$, то в силу (12)

$$
L_{n+1}^{\alpha-1}(x)=L_{n+1}^{-1}(x)=-\frac{x}{n+1} L_{n}^{1}(x),
$$

и, стало быть,

$$
\left(\left|L_{n}^{\alpha+1}(x) L_{n+1}^{\alpha-1}(x)\right|\right)^{1 / 2}=\left(\frac{x}{n+1}\right)^{1 / 2}\left|L_{n}^{1}(x)\right| .
$$

Если $0 \leqslant x \leqslant 12 n \ln 7$, то из (13) непосредственно вытекает оценка

$$
A_{n}^{1}(x)\left(\frac{x}{n+1}\right)^{1 / 2} \leqslant c(\alpha) A_{n}^{0}(x)
$$


поэтому в силу (57) и (14)

$$
\left(\left|L_{n}^{\alpha+1}(x) L_{n+1}^{\alpha-1}(x)\right|\right)^{1 / 2} \leqslant c(\alpha)\left(\frac{x}{n+1}\right)^{1 / 2} A_{n}^{1}(x) \leqslant c(\alpha) A_{n}^{0}(x) .
$$

Покажем, что оценка (58) остается в силе также для $x \geqslant(12 \ln 7) n$. Воспользуемся формулой (8) и интегральной формулой Коши. Тогда при $x>0$ имеем

$$
\left|L_{n}^{1}(x)\right|=\frac{1}{2 \pi}\left|\int_{\gamma} \frac{e^{x-t} t^{n+1}}{x(x-t)^{n+1}} d t\right|,
$$

где $\gamma$ - замкнутьй контур, охватывающий точку $t=x$. Составим контур $\gamma$ из отрезка $t=5 x / 6+i \tau\left(\tau_{-1} \leqslant \tau \leqslant \tau_{1}\right)$ и дуги окружности $|t|=7 x / 6$, где $\tau_{-1}$ и $\tau_{1}-$ точки пересечения прямой $t=5 x / 6+i \tau$ и окружности $|t|=7 x / 6$. Тогда из (59) имеем

$$
\left|L_{n}^{1}(x)\right| \leqslant \frac{7}{12 \pi} \int_{\gamma}\left|e^{x-t}\left(\frac{t}{x-t}\right)^{n}\right| \frac{|d t|}{|x-t|} \leqslant \frac{7^{n+2}}{6} e^{x / 6}=\frac{49}{6} 7^{n} e^{-x / 12} e^{x / 4} .
$$

Пусть $x \geqslant(12 \ln 7) n$, тогда

$$
\left(\frac{x}{n+1}\right)^{1 / 2} 7^{n} e^{-x / 12}=\exp \left(\frac{(12 \ln 7) n-x}{12}+\frac{1}{2} \ln \frac{x}{n+1}\right) \leqslant c .
$$

Из (60) и (61) находим

$$
\left(\frac{x}{n+1}\right)^{1 / 2}\left|L_{n}^{1}(x)\right| \leqslant c e^{x / 4}=c A_{n}^{0}(x), \quad x \geqslant(12 \ln 7) n .
$$

Сопоставляя (57) и (62), убеждаемся в справедливости оценки (58). Другими словами, оценка (56) верна также при $\alpha=0$.

Утверждение теоремы 7.1 вытекает из (54)-(56).

В заключение автор выражает признательность Б. С. Кашину за обсуждение результатов и ценные замечания.

\section{СПИСОК ЦИТИРОВАННОЙ ЛИТЕРАТУРЫ}

[1] Бейтмен Г., Эрдейи А. Высшие трансцендентные функции. Т. 2. М.: Наука, 1974.

[2] Шарапудинов И. И. Асимптотические свойства многочленов Мейкснера и их приложения // Деп. ВИНИТИ. № 1433-83.

[3] Шарапудинов И. И. Некоторые свойства ортогональных многочленов Мейкснера // Матем. заметки. 1990. Т. 47. №3. С. 135-137.

[4] Шарапудинов И.И. Некоторые вопросы теории ортогональных систем. Дисс. ... д. ф.-м. н. М.: МИАН, 1991.

[5] Sharapudinov I. I. Asymptotics of Meixner's polynomials // Facta Univ. Ser. Math. Inform. 1991. V. 6. P. 13-21.

[6] Askey R., Wainger S. Mean convergence of expansions in Laguerre and Hermite series // Amer. J. Math. 1965. V. 87. P. 695-708.

[7] Muckenhoupt B. Mean convergence of Hermite and Laguerre series // Trans. Amer. Math. Soc. 1970. V. 147. P. 433-460.

[8] Сегё Г. Ортогональные многочлены. М.: Физматгиз, 1962.

[9] Шарапудинов И. И. Асимптотические свойства ортогональных многочленов Хана дискретной переменной // Матем. сб. 1989. Т. 180. № 9. С. 1259-1277.

Дагестанский государственный педагогический университет,

Поступило г. Махачкала

28.03 .96

Исправленный вариант 